\title{
Mode structure of a broadband high gain parametric amplifier
}

\author{
Xin Chen, Jacob Zhang, and Z. Y. Ou (1)* \\ Department of Physics, Indiana University-Purdue University Indianapolis, Indianapolis, Indiana 46202, USA
}

(Received 3 March 2021; revised 14 May 2021; accepted 19 May 2021; published 4 June 2021)

\begin{abstract}
A high gain parametric amplifier with a single-pass pulsed pump is known to generate broadband twin photon fields that are entangled in amplitude and phase but have complicated spectral correlation. Fortunately, they can be decomposed into independent temporal modes. However, the early treatment of the parametric interaction Hamiltonian at strong pumping does not consider the issue of the time-ordering problem of the interaction Hamiltonian and thus leads to the inaccurate conclusion that the mode structure and the temporal mode functions do not change as the gain increases. Recent studies have revealed the change of the spectrum with the gain of the process. In this paper we use an approach that is usually employed for treating nonlinear interferometers and avoids the time-ordering issue. This allows us to derive an evolution equation in differential-integral form. Numerical solutions for the high gain situation indicate a gain-dependent mode structure that has its mode distributions changed and mode functions broadened as the gain increases. This study will enable us to have a complete picture of the mode structure of parametric processes and produce high quality quantum sources for a variety of applications of quantum technology.
\end{abstract}

DOI: 10.1103/PhysRevResearch.3.023186

\section{INTRODUCTION}

Parametric processes in the high gain regime are the most common and simple processes for generating a variety of quantum states including twin beam states, squeezed states, Einstein-Podolsky-Rosen (EPR) entangled states [1] for the applications [2] in continuous variable quantum computing [3-6] and information processing, quantum communication [7,8], and quantum metrology [9]. For achieving high gain operation, ultrashort pulse pumping in single-pass configuration [10] is usually preferred due to its high concentration of energy and ease of operation. This has been done in optical waveguide structure [11] and optical fiber systems [12] in which spatial modes are well defined. However, due to ultrashort pulses in pumping and dispersion in nonlinear media, the spectral correlation is extremely complicated [13].

Fortunately, the complicated spectral correlation can be decomposed into independent temporal modes [14,15]. This was first pointed out by Law et al. [16] for the low gain case where two-photon events dominate and used in the analysis of the mode structure for the generation of high quality single- and two-photon states [17]. In the high gain regime, the existence of independent pairwise entangled temporal modes was recently confirmed experimentally in a direct measurement of the temporal mode profiles and in subsequent

\footnotetext{
*Present address: Department of Physics, City University of Hong Kong, 83 Tat Chee Avenue, Kowloon, Hong Kong, People's Republic of China; zou@iupui.edu

Published by the American Physical Society under the terms of the Creative Commons Attribution 4.0 International license. Further distribution of this work must maintain attribution to the author(s) and the published article's title, journal citation, and DOI.
}

correlation measurement [18]. However, the early treatment of parametric interaction Hamiltonian $[15,19]$ only works in the limit of low gain or in the regime of spontaneous emission but fails at high gain because it does not consider the issue of time ordering of the Hamiltonian [20] and thus leads to the inaccurate result that temporal modes do not change in the high gain limit [15]. Indeed, recent studies with approaches that avoid the time-ordering issue have shown the spectrum broadening as the gain increases [21,22]. It should be pointed out that the treatment by Wasilewski et al. [14] also avoided the time-ordering issue by quantizing a classical propagation equation. A similar approach was adopted by Reddy et al. [23] in treating broadband frequency conversion process. The experiment that directly measured the temporal mode functions also confirmed the change of mode structure and mode functions as the gain increases [18].

The change of mode structure and mode functions with gain is troublesome in the production and applications of high quality quantum sources with quantum entanglement and noise reduction such as EPR entangled states and squeezed states, which require high gain operation in parametric processes. This is because the measurement on these states relies on the homodyne measurement technique in which the mode match between the local oscillator (LO) field and the quantum field is paramount and any mode mismatch is equivalent to losses and introduces extra vacuum noise. Knowledge of the exact profile of the mode functions will enable us to tailor the shape of the LO field to match the quantum field [18]. However, the change of the mode functions means that we also need to adjust the shape of the LO to accommodate the change. The shape of the mode functions is also important for quantum pulse gates [24-26] in temporal mode multiplexing.

So far an analysis of how mode structure and mode functions change with the gain is lacking. In this paper we 


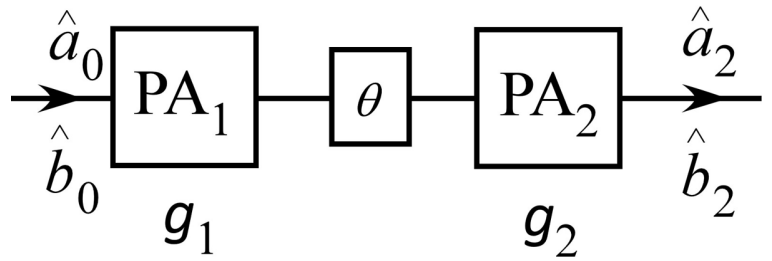

FIG. 1. An SU(1,1) interferometer with parametric amplifiers $\mathrm{PA}_{1}$ and $\mathrm{PA}_{2}$ in the place of beam splitters.

investigate the pulse-pumped single-pass parametric processes at arbitrary pumping power. We use an operator input-output approach that is usually employed for treating multistage nonlinear interferometers [27]. This avoids the time-ordering issue of the interaction Hamiltonian and allows us to derive a set of coupled operator evolution equations in differential-integral form. We solve them numerically and analyze the mode structure and mode functions at the final output ports as a function of the pump parameter.

The paper is organized as follows. In Sec. II we introduce the input-output approach for the single-mode case of a multistage nonlinear interferometer involving parametric processes. In Sec. III we apply the approach to the evolution of the fields in broadband parametric processes pumped by a pulse for an arbitrary length of nonlinear medium. We discuss mode decomposition in Sec. IV and solve numerically the state evolution in Sec. V to find how mode structure and mode functions change with the gain. We conclude with a discussion in Sec. VI.

\section{MULTISTAGE SU(1,1) INTERFEROMETERS}

In order to reveal the issue of time ordering in the derivation of the evolution operator in parametric processes and find ways to tackle it, we consider an $\mathrm{SU}(1,1)$ interferometer, shown in Fig. 1, which consists of two parametric amplifiers $\left(\mathrm{PA}_{1}\right.$ and $\left.\mathrm{PA}_{2}\right)$ characterized by gain parameters $g_{1}$ and $g_{2}$ together with a phase shift $\theta$ in between. This interferometer has recently been studied extensively [28] for precision phase measurement beyond the standard quantum limit [29], quantum imaging with undetected photons [30], and quantum state engineering [31,32].

The two PAs are described by the Hamiltonians

$$
\hat{H}_{\mathrm{PA}}\left(\xi_{j}\right)=i \hbar \xi_{j} \hat{a}^{\dagger} \hat{b}^{\dagger}-i \hbar \xi_{j}^{*} \hat{a} \hat{b}
$$

where $j=1,2$. The input-output relations can be derived from evolution operators $\hat{U}_{j}=e^{(1 / i \hbar) \hat{H}_{\mathrm{PA}}\left(\xi_{j}\right) t}(j=1,2)$ and are given, respectively, as

$$
\begin{array}{ll}
\hat{a}_{1}=G_{1} \hat{a}_{0}+g_{1} \hat{b}_{0}^{\dagger}, & \hat{b}_{1}=G_{1} \hat{b}_{0}+g_{1} \hat{a}_{0}^{\dagger}, \\
\hat{a}_{2}=G_{2} \hat{a}_{1}^{\prime}+g_{2} \hat{b}_{1}^{\prime \dagger}, & \hat{b}_{2}=G_{2} \hat{b}_{1}^{\prime}+g_{2} \hat{a}_{1}^{\prime \dagger},
\end{array}
$$

where $\hat{a}_{1}^{\prime}=\hat{a}_{1} e^{i \theta / 2}, \hat{b}_{1}^{\prime}=\hat{b}_{1} e^{i \theta / 2}$, the amplitude gains $g_{j} \equiv$ $\left(\xi_{j} /\left|\xi_{j}\right|\right) \sinh \left|\xi_{j} t\right|(j=1,2)$, and $G_{j}=\cosh \left|\xi_{j} t\right|$ for an interaction time period of $t$. Here we assume that the phase shifts are the same for both fields: $\theta_{a}=\theta_{b}=\theta / 2$. The outputs of the interferometer are then [27]

$$
\hat{a}_{2}=G_{T} \hat{a}_{0}+g_{T} \hat{b}_{0}^{\dagger}, \quad \hat{b}_{2}=G_{T} \hat{b}_{0}+g_{T} \hat{a}_{0}^{\dagger},
$$

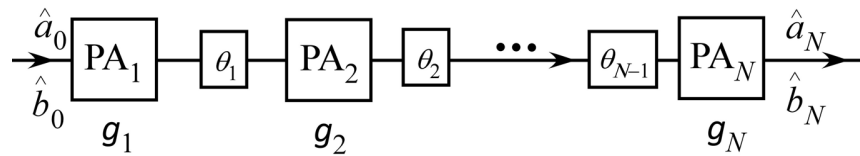

FIG. 2. Multistage SU(1,1) interferometer.

with

$$
\begin{aligned}
G_{T} & =G_{1} G_{2} e^{i \theta / 2}+g_{1}^{*} g_{2} e^{-i \theta / 2}, \\
g_{T} & =G_{1}^{*} g_{2} e^{-i \theta / 2}+g_{1} G_{2} e^{i \theta / 2} .
\end{aligned}
$$

This shows that we can treat the whole system as one parametric amplifier with equivalent amplitude gains $g_{T}$ and $G_{T}$. Furthermore, besides a propagation phase of $e^{i \theta / 2}$ for both fields, the extra phase shift $e^{i \theta}$ can be absorbed in $g_{2}$ by redefining $g_{2}^{\prime} \equiv g_{2} e^{-i \theta}$ or $\xi_{2}^{\prime} \equiv \xi_{2} e^{-i \theta}$. Here $\xi_{2}^{\prime}=\xi_{2} e^{-i \theta}$ takes the propagation phase shift $e^{i \theta}$ into consideration. Notice that only when $\arg \xi_{1}=\arg \xi_{2}-\theta$, other than a common phase of $e^{i \theta / 2}$ for both fields, can the whole system be described by an equivalent overall Hamiltonian $\hat{H}_{T}=$ $\hat{H}_{\mathrm{PA}}\left(\xi_{T}\right)=\hat{H}_{\mathrm{PA}}\left(\xi_{1}\right)+\hat{H}_{\mathrm{PA}}\left(\xi_{2}^{\prime}\right)$ with $\xi_{T}=\xi_{1}+\xi_{2}^{\prime}$. However, if $\arg \xi_{1} \neq \arg \xi_{2}-\theta$, then $\hat{H}_{T} \neq \hat{H}_{\mathrm{PA}}\left(\xi_{1}\right)+\hat{H}_{\mathrm{PA}}\left(\xi_{2}^{\prime}\right)$. This is because $e^{(1 / i \hbar) \hat{H}_{\mathrm{PA}}\left(\xi_{1}\right) t} e^{(1 / i \hbar) \hat{H}_{\mathrm{PA}}\left(\xi_{2}^{\prime}\right) t} \neq e^{(1 / i \hbar)\left[\hat{H}_{\mathrm{PA}}\left(\xi_{1}\right)+\hat{H}_{\mathrm{PA}}\left(\xi_{2}^{\prime}\right)\right] t}$ if $\left[\hat{H}_{\mathrm{PA}}\left(\xi_{1}\right), \hat{H}_{\mathrm{PA}}\left(\xi_{2}^{\prime}\right)\right] \neq 0$ when $\arg \xi_{1} \neq \arg \xi_{2}-\theta$.

This will have an inconvenient consequence when we extend the interferometer to multiple stages [17,32], as shown in Fig. 2. Because the phases in each stage are arbitrary, we therefore cannot write the overall Hamiltonian as the sum of each stage

$$
\hat{H}_{T} \neq \sum_{j} i \hbar\left(\xi_{j}^{\prime} \hat{a}^{\dagger} \hat{b}^{\dagger}-\xi_{j}^{*} \hat{a} \hat{b}\right),
$$

where the phase shifts at each stage are absorbed in the interaction parameter $\xi_{j}^{\prime}=\xi_{j} e^{-i \theta_{j}}$.

To solve this problem, we can proceed by using repeatedly Eqs. (4) to add each stage and obtain a recursive relation. Specifically for Eqs. (4), we treat all the stages added up to stage $k$ as the first PA with equivalent amplitude gains $G_{T}(k)$ and $g_{T}(k)$ and the second PA is the $(k+1)$ th stage to be added:

$$
\begin{aligned}
& G_{T}(k+1)=G_{T}(k) G_{k+1} e^{i \theta_{k} / 2}+g_{T}^{*}(k) g_{k+1} e^{-i \theta_{k} / 2}, \\
& g_{T}(k+1)=G_{T}^{*}(k) g_{k+1} e^{-i \theta_{k} / 2}+g_{T}(k) G_{k+1} e^{i \theta_{k} / 2} .
\end{aligned}
$$

Unfortunately, we cannot find an analytical expression for the final outputs. In order to have some general idea about the outputs, we consider that each stage has an infinitesimally small gain and phase shift whose sizes are proportional to an infinitesimal length scale $\Delta x$ along the field propagation direction: $g_{k} \approx \zeta(x) \Delta x, \theta_{k} \approx \eta(x) \Delta x$, and we use the location $x=k \Delta x$ to denote the $k$ th stage. When $\Delta x \rightarrow 0, G_{k+1}=$ $\sqrt{1+\left|g_{k+1}\right|^{2}} \approx 1+o(\Delta x)$. So Eqs. (6) can be approximated as

$$
\begin{aligned}
& G_{T}(x+\Delta x) \approx G_{T}(x)\left(1+\frac{i \eta \Delta x}{2}\right)+g_{T}^{*}(x) \zeta \Delta x, \\
& g_{T}(x+\Delta x) \approx G_{T}^{*}(x) \zeta \Delta x+g_{T}(x)\left(1+\frac{i \eta \Delta x}{2}\right)
\end{aligned}
$$


or

$$
\begin{aligned}
\frac{d}{d x} G_{T}(x) & =\zeta(x) g_{T}^{*}(x)+\frac{i \eta(x)}{2} G_{T}(x), \\
\frac{d}{d x} g_{T}(x) & =\zeta(x) G_{T}^{*}(x)+\frac{i \eta(x)}{2} g_{T}(x) .
\end{aligned}
$$

These are the evolution equations for a parametric amplifier with continuous gain function $\zeta(x)$. The phase parameter $\eta(x)$ usually corresponds to phase mismatching. The initial condition is obviously $G_{T}(0)=1$ and $g_{T}(0)=0$. It is hard to solve analytically the differential equations if $\zeta(x)$ and $\eta(x)$ depend on location $x$. For simplicity, let us assume that $\zeta$ and $\eta$ are constant. Then Eqs. (8) can be solved analytically and have the following solution. If $\zeta \leqslant \eta / 2$ and $\eta_{0} \equiv \sqrt{\eta^{2}-4|\zeta|^{2}}$, we have

$$
\begin{aligned}
& G_{T}(x)=\cos \left(\frac{\eta_{0} x}{2}\right)+i \frac{\eta}{\eta_{0}} \sin \left(\frac{\eta_{0} x}{2}\right), \\
& g_{T}(x)=\frac{2 \zeta}{\eta_{0}} \sin \left(\frac{\eta_{0} x}{2}\right) .
\end{aligned}
$$

If $\zeta \geqslant \eta / 2$ and $\zeta_{0} \equiv \sqrt{|\zeta|^{2}-(\eta / 2)^{2}}$, we have

$$
\begin{aligned}
& G_{T}(x)=\cosh \left(\zeta_{0} x\right)+i \frac{\eta}{2 \zeta_{0}} \sinh \left(\zeta_{0} x\right), \\
& g_{T}(x)=\frac{\zeta}{\zeta_{0}} \sinh \left(\zeta_{0} x\right) .
\end{aligned}
$$

The exponential growth of the gain when $\zeta \geqslant \eta / 2$ is typical of high gain parametric amplifiers. However, the oscillatory low gain behavior when $\zeta \leqslant \eta / 2$ is the result of interference, as we will see in the following.

In the limit of $\zeta \ll 1$, we have

$$
\begin{aligned}
G_{T}(x) & =e^{i \eta x / 2} \\
g_{T}(x) & =\zeta x \operatorname{sinc}\left(\frac{\eta x}{2}\right) \equiv e^{i \eta x / 2} x \xi_{T} \\
& =e^{i \eta x / 2} \int_{0}^{x} d x^{\prime} \zeta e^{-i \eta x^{\prime}} .
\end{aligned}
$$

The extra phase factor $e^{i \eta x / 2}$ extracted out of $g_{T}$ is for the consistency with $G_{T}$ and is due to propagation of the fields through the system. The gain parameters in Eq. (11) are equivalent to an overall Hamiltonian of the interaction parameter $\xi_{T} \equiv \zeta e^{-i \eta x / 2} \operatorname{sinc}(\eta x / 2)$ with evolution time $t$ replaced by $x$,

$$
\hat{H}_{T}=i \hbar\left(\xi_{T} \hat{a}^{\dagger} \hat{b}^{\dagger}-\xi_{T}^{*} \hat{a} \hat{b}\right),
$$

which can thought of as the sum of all the stages:

$$
\hat{H}_{T}=\frac{1}{x} \int_{0}^{x} d x^{\prime} i \hbar \zeta e^{-i \eta x^{\prime}} \hat{a}^{\dagger} \hat{b}^{\dagger}+\text { H.c. }=\frac{1}{x} \int_{0}^{x} \hat{H}\left(d \xi^{\prime}\right) .
$$

Here $d \xi^{\prime}=\zeta e^{-i \eta x^{\prime}} d x^{\prime}$ is the infinitesimal gain parameter for each infinitesimal stage. Note that this equivalence is true only when $\zeta(x)=$ const and $\eta(x)=$ const.

As a matter of fact, when the gain parameters $\left|g_{1}\right|,\left|g_{2}\right| \ll$ 1, we can add the two Hamiltonian to obtain the overall Hamiltonian: $\hat{H}_{T}=\hat{H}_{\mathrm{PA}}\left(\xi_{T}\right)=\hat{H}_{\mathrm{PA}}\left(\xi_{1}\right)+\hat{H}_{\mathrm{PA}}\left(\xi_{2}^{\prime}\right)$. This can be seen from the evolution operator

$$
\begin{aligned}
\hat{U}_{T} & =\hat{U}_{2}\left(\xi_{2}\right) \hat{U}_{1}\left(\xi_{1}^{\prime}\right) \\
& =e^{\hat{H}\left(\xi_{2}\right) \Delta x / i \hbar} e^{\hat{H}\left(\xi_{1}^{\prime}\right) \Delta x / i \hbar} \\
& \approx\left[1+\hat{H}\left(\xi_{2}\right) \Delta x / i \hbar\right]\left[1+\hat{H}\left(\xi_{1}^{\prime}\right) \Delta x / i \hbar\right] \\
& \approx 1+\hat{H}\left(\xi_{2}\right) \Delta x / i \hbar+\hat{H}\left(\xi_{1}^{\prime}\right) \Delta x / i \hbar \\
& =1+\hat{H}_{T} \Delta x / i \hbar \\
& \approx e^{\hat{H}_{T} \Delta x / i \hbar} .
\end{aligned}
$$

Here we have assumed $\left|g_{1}\right|=\left|\xi_{1}\right| \Delta x \ll 1,\left|g_{2}\right|=\left|\xi_{1}^{\prime}\right| \Delta x \ll$ 1 , and $\xi_{1}^{\prime}=\xi_{1} e^{-i \theta}$ with phase shift $e^{-i \theta}$ included. So when the amplitude gains $\left|g_{j}\right| \ll 1$, we can simply add the Hamiltonian of each stage:

$$
\hat{H}_{T}=\sum_{j} i \hbar\left(\xi_{j}^{\prime} \hat{a}^{\dagger} \hat{b}^{\dagger}-\xi_{j}^{*} \hat{a} \hat{b}\right)
$$

and

$$
g_{T}=\sum_{k} g_{k}^{\prime}=\sum_{k} g_{k} e^{-i \theta_{k}}
$$

The above can also be thought of as a result of two-photon interference between the pair of photons generated by each stage $[17,32]$. This can be confirmed by looking at the output state for the vacuum input

$$
|\Psi\rangle_{T}=\hat{U}_{T}|0\rangle \approx|0\rangle+\left(\sum_{k} g_{k}^{\prime}\right)\left|1_{a}, 1_{b}\right\rangle=|0\rangle+\sum_{k}|\Psi\rangle_{k},
$$

with $|\Psi\rangle_{k}$ as the two-photon state generated by the $k$ th stage.

Notice that Eq. (15) is true only if the overall amplitude gain $g_{T}$ is much smaller than 1 so that the last step of Eq. (14) stands. For the high gain case, Eq. (15) does not stand and we have to resort to Eqs. (6) or (10). This will pose a serious problem in finding solution for a broadband parametric amplifier in the high gain regime.

\section{BROADBAND PARAMETRIC AMPLIFIER}

When parametric processes are pumped by high power pulses, broadband parametric amplification is achieved. They can be used to produce quantum entangled fields with a wide bandwidth. The traditional treatment of this situation is to start with a multimode Hamiltonian of the form [13,15,19]

$$
\begin{aligned}
\hat{H}_{M}= & \chi \int d \omega_{1} d \omega_{2} d \omega_{3} \Psi\left(\omega_{1}, \omega_{2}, \omega_{3}\right) \hat{a}^{\dagger}\left(\omega_{1}\right) \hat{b}^{\dagger}\left(\omega_{2}\right) \\
& \times A_{p}\left(\omega_{3}\right) e^{i\left(\omega_{1}+\omega_{2}-\omega_{3}\right) t}+\text { H.c. },
\end{aligned}
$$

where the subscript $M$ denotes the multimode, $\chi$ is some parameter proportional to the nonlinear coefficient of the nonlinear medium of length $L_{0}, A_{p}\left(\omega_{3}\right)$ is the spectral amplitude of the pump field, and $\Psi\left(\omega_{1}, \omega_{2}, \omega_{3}\right)$ is obtained from spatial integration

$$
\Psi\left(\omega_{1}, \omega_{2}, \omega_{3}\right) \equiv \int_{0}^{L_{0}} d z e^{-i z \Delta k}=L_{0} \frac{\sin \beta}{\beta} e^{-i \beta},
$$


with $\beta \equiv \Delta k L_{0} / 2$ and $\Delta k \equiv k_{1}+k_{2}-k_{3}$ as the phase mismatching. We then find the evolution operator as

$$
\hat{U}=\exp \left\{\frac{1}{i \hbar} \int_{-\infty}^{\infty} d t \hat{H}_{M}\right\},
$$

where time integration gives rise to a delta function $\delta\left(\omega_{1}+\right.$ $\left.\omega_{2}-\omega_{3}\right)$ and the integrated Hamiltonian has the form

$\int d t \hat{H}_{M}=i \hbar G \int d \omega_{1} d \omega_{2} \Phi\left(\omega_{1}, \omega_{2}\right) \hat{a}^{\dagger}\left(\omega_{1}\right) \hat{b}^{\dagger}\left(\omega_{2}\right)+$ H.c.

with $G \equiv \chi / C$ as a dimensionless gain parameter such that

$$
\Phi\left(\omega_{1}, \omega_{2}\right) \equiv 2 \pi C L_{0} \frac{\sin \beta}{\beta} e^{-i \beta} A_{p}\left(\omega_{1}+\omega_{2}\right)
$$

is normalized: $\int d \omega_{1} d \omega_{2}\left|\Phi\left(\omega_{1}, \omega_{2}\right)\right|^{2}=1$. In general, this gives rise to a complicated coupling of different frequency components at the outputs,

$$
\begin{aligned}
\hat{a}^{(o)}\left(\omega_{1}\right)= & \hat{U}^{\dagger} \hat{a}\left(\omega_{1}\right) \hat{U} \\
= & \int h_{1 a}\left(\omega_{1}, \omega_{1}^{\prime}\right) \hat{a}\left(\omega_{1}^{\prime}\right) d \omega_{1}^{\prime} \\
& +\int h_{2 a}\left(\omega_{1}, \omega_{2}^{\prime}\right) \hat{b}^{\dagger}\left(\omega_{2}^{\prime}\right) d \omega_{2}^{\prime}, \\
\hat{b}^{(o)}\left(\omega_{2}\right)= & \hat{U}^{\dagger} \hat{b}\left(\omega_{2}\right) \hat{U} \\
= & \int h_{1 b}\left(\omega_{2}, \omega_{2}^{\prime}\right) \hat{b}\left(\omega_{2}^{\prime}\right) d \omega_{2}^{\prime} \\
& +\int h_{2 b}\left(\omega_{1}^{\prime}, \omega_{2}\right) \hat{a}^{\dagger}\left(\omega_{1}^{\prime}\right) d \omega_{1}^{\prime},
\end{aligned}
$$

where $\hat{a}^{(o)}$ and $\hat{b}^{(o)}$ are the outputs at the end of nonlinear medium and $\hat{a}\left(\omega_{1}\right)$ and $\hat{b}\left(\omega_{2}\right)$ are those at the start. However, we can make a singular value decomposition of the joint spectrum function $\Phi\left(\omega_{1}, \omega_{2}\right)$,

$$
\Phi\left(\omega_{1}, \omega_{2}\right)=\sum_{k} r_{k} \psi_{k}\left(\omega_{1}\right) \varphi_{k}\left(\omega_{2}\right),
$$

where $\left\{\psi_{k}, \phi_{k}\right\}$ are two sets of orthonormal functions $\int d \omega_{1} \psi_{j}^{*}\left(\omega_{1}\right) \psi_{k}\left(\omega_{1}\right)=\delta_{j k}=\int d \omega_{2} \varphi_{j}^{*}\left(\omega_{2}\right) \varphi_{k}\left(\omega_{2}\right)$ and $\left\{r_{k}\right\}$ are non-negative numbers satisfying $\sum_{k} r_{k}^{2}=1$. Then Eq. (21) becomes

$$
\int d t \hat{H}_{M}=i \hbar G \sum_{k} r_{k} \hat{A}_{k}^{\dagger} \hat{B}_{k}^{\dagger}+\text { H.c., }
$$

with $\hat{A}_{k} \equiv \int d \omega_{1} \psi_{j}^{*}\left(\omega_{1}\right) \hat{a}\left(\omega_{1}\right)$ and $\hat{B}_{k} \equiv \int d \omega_{2} \varphi_{j}^{*}\left(\omega_{2}\right) \hat{b}\left(\omega_{2}\right)$ satisfying $\left[\hat{A}_{j}, \hat{A}_{k}^{\dagger}\right]=\delta_{j k}=\left[\hat{B}_{j}, \hat{B}_{k}^{\dagger}\right]$. Together with Eq. (20), this leads to decoupling of the different temporal modes $\hat{A}_{k}$ and $\hat{B}_{k}$ for the two output fields:

$$
\begin{aligned}
& \hat{A}_{k}^{(o)}=\cosh \left(r_{k} G\right) \hat{A}_{k}+\sinh \left(r_{k} G\right) \hat{B}_{k}^{\dagger}, \\
& \hat{B}_{k}^{(o)}=\cosh \left(r_{k} G\right) \hat{B}_{k}+\sinh \left(r_{k} G\right) \hat{A}_{k}^{\dagger} .
\end{aligned}
$$

Unfortunately, it was pointed out [20] that the evolution operator in Eq. (20) is not correct for the Hamiltonian (18) because $\left[\hat{H}_{M}(t), \hat{H}_{M}\left(t^{\prime}\right)\right] \neq 0$ for $t \neq t^{\prime}$. The reason is the same as those for Eq. (1); however, Eqs. (23) and (23) are still correct for the Hamiltonian (18).

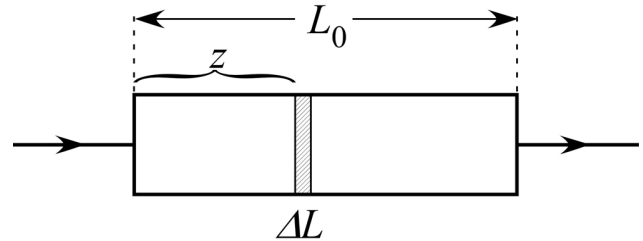

FIG. 3. Parametric amplifier from a single-pass pulse-pumped nonlinear medium.

In order to treat this in a correct manner, we can apply the same approach as in the preceding section. Consider a nonlinear medium of length $L$ which is divided into a small segment of size $\Delta L$, as shown in Fig. 3. Let us treat the small segment first. The Hamiltonian is given from Eq. (18) for the small segment as

$$
\begin{aligned}
\hat{H}(z, \Delta L)= & \chi \int d \omega_{1} d \omega_{2} d \omega_{3} \int_{z}^{z+\Delta L} d z^{\prime} e^{-i z^{\prime} \Delta k} \hat{a}^{\dagger}\left(\omega_{1}, z\right) \\
& \times \hat{b}^{\dagger}\left(\omega_{2}, z\right) A_{p}\left(\omega_{3}\right) e^{i\left(\omega_{1}+\omega_{2}-\omega_{3}\right) t}+\text { H.c., }
\end{aligned}
$$

where the spatial integration starts at $z$ instead of 0 because the $\Delta L$ segment is located at $z$ inside the medium and we assume that there is no pump depletion. For $\Delta L=d L \rightarrow 0$, we have

$$
\begin{aligned}
\hat{H}(z, d L)= & \chi d L \int d \omega_{1} d \omega_{2} d \omega_{3} e^{-i z \Delta k} \hat{a}^{\dagger}\left(\omega_{1}, z\right) \hat{b}^{\dagger}\left(\omega_{2}, z\right) \\
& \times A_{p}\left(\omega_{3}\right) e^{i\left(\omega_{1}+\omega_{2}-\omega_{3}\right) t}+\text { H.c. }+o(d L) .
\end{aligned}
$$

The evolution operator for this segment is given by the Dyson series

$$
\hat{U}(z, \Delta L)=1+\sum_{n=1} \hat{U}_{n}
$$

with

$$
\begin{aligned}
\hat{U}_{n}= & \left(\frac{1}{i \hbar}\right)^{n} \int_{-\infty}^{\infty} d t_{1} \int_{-\infty}^{t_{1}} d t_{2} \cdots \int_{-\infty}^{t_{n-1}} d t_{n} \\
& \times \hat{H}\left(z, d L, t_{1}\right) \cdots \hat{H}\left(z, d L, t_{n}\right) .
\end{aligned}
$$

Equation (29) becomes Eq. (20) if $\left[\hat{H}\left(z, d L, t_{1}\right)\right.$, $\left.\hat{H}\left(z, d L, t_{2}\right)\right]=0$, but it is not true for $\hat{H}(z, d L)$ in Eq. (28). On the other hand, for $d L \rightarrow 0$ we have [19]

$$
\hat{U}(z, d L)=1+\hat{U}_{1}+o(d L) \approx 1+\int d t \hat{H}(z, d L)
$$

So we obtain the evolution of the field operators

$$
\begin{aligned}
\hat{a}\left(\omega_{1}, z+d L\right)= & \hat{U}^{\dagger}(z, d L) \hat{a}\left(\omega_{1}, z\right) \hat{U}(z, d L) \\
= & \hat{a}\left(\omega_{1}, z\right)+\left[\hat{a}\left(\omega_{1}, z\right), \int d t \hat{H}(z, d L)\right] \\
= & \hat{a}\left(\omega_{1}, z\right)+2 \pi \chi d L \int d \omega_{2} \hat{b}^{\dagger}\left(\omega_{2}, z\right) \\
& \times e^{-i z \Delta k} A_{p}\left(\omega_{1}+\omega_{2}\right),
\end{aligned}
$$

where the time integral gives rise to a $\delta$ function for $\omega_{1}+\omega_{2}-\omega_{3}$ and $\Delta k=\Delta k_{\mid \omega_{3}=\omega_{1}+\omega_{2}}$. With $d \hat{a}\left(\omega_{1}, z\right) / d z=$ $\left[\hat{a}\left(\omega_{1}, z+d L\right)-\hat{a}\left(\omega_{1}, z\right)\right] / d L$ we have

$$
\frac{d}{d z} \hat{a}\left(\omega_{1}, z\right)=2 \pi \chi \int d \omega_{2} e^{-i z \Delta k} A_{p}\left(\omega_{1}+\omega_{2}\right) \hat{b}^{\dagger}\left(\omega_{2}, z\right) .
$$


Likewise, we obtain

$$
\frac{d}{d z} \hat{b}\left(\omega_{2}, z\right)=2 \pi \chi \int d \omega_{1} e^{-i z \Delta k} A_{p}\left(\omega_{1}+\omega_{2}\right) \hat{a}^{\dagger}\left(\omega_{1}, z\right) .
$$

Note that Eqs. (33) and (34) are similar in form to those derived in Refs. [21,22] with different methods. Using the input-output relation in Eq. (23) at location $z$, we obtain

$$
\begin{aligned}
& \frac{d}{d z} h_{1 a}\left(\omega_{1}, \omega_{1}^{\prime}, z\right)=\int d \omega_{2} f\left(\omega_{1}, \omega_{2}\right) h_{2 b}^{*}\left(\omega_{1}^{\prime}, \omega_{2}, z\right), \\
& \frac{d}{d z} h_{2 b}\left(\omega_{1}^{\prime}, \omega_{2}, z\right)=\int d \omega_{1} f\left(\omega_{1}, \omega_{2}\right) h_{1 a}^{*}\left(\omega_{1}, \omega_{1}^{\prime}, z\right),
\end{aligned}
$$

and

$$
\begin{aligned}
& \frac{d}{d z} h_{1 b}\left(\omega_{2}, \omega_{2}^{\prime}, z\right)=\int d \omega_{1} f\left(\omega_{1}, \omega_{2}\right) h_{2 a}^{*}\left(\omega_{1}, \omega_{2}^{\prime}, z\right), \\
& \frac{d}{d z} h_{2 a}\left(\omega_{1}, \omega_{2}^{\prime}, z\right)=\int d \omega_{2} f\left(\omega_{1}, \omega_{2}\right) h_{1 b}^{*}\left(\omega_{2}, \omega_{2}^{\prime}, z\right),
\end{aligned}
$$

where $f\left(\omega_{1}, \omega_{2}\right) \equiv 2 \pi \chi e^{-i z \Delta k} A_{p}\left(\omega_{1}+\omega_{2}\right)$. Since we have $\hat{a}^{(o)}\left(\omega_{1}, z=0\right)=\hat{a}\left(\omega_{1}\right)$ and $\hat{b}^{(o)}\left(\omega_{2}, z=0\right)=\hat{b}\left(\omega_{2}\right)$ at the start, the initial conditions are

$$
\begin{aligned}
& h_{1 a}\left(\omega_{1}, \omega_{1}^{\prime}, z=0\right)=\delta\left(\omega_{1}-\omega_{1}^{\prime}\right), \\
& h_{1 b}\left(\omega_{2}, \omega_{2}^{\prime}, z=0\right)=\delta\left(\omega_{2}-\omega_{2}^{\prime}\right), \\
& h_{2 a}\left(\omega_{1}, \omega_{2}^{\prime}, z=0\right)=0, \\
& h_{2 b}\left(\omega_{2}, \omega_{1}^{\prime}, z=0\right)=0 .
\end{aligned}
$$

From Eqs. (35) and (36) and the initial conditions (37), we can verify that

$$
\begin{aligned}
& \int d \omega_{1}^{\prime} h_{1 a}\left(\omega_{1}, \omega_{1}^{\prime}, z\right) h_{1 a}^{*}\left(\bar{\omega}_{1}, \omega_{1}^{\prime}, z\right) \\
& -\int d \omega_{2}^{\prime} h_{2 a}\left(\omega_{1}, \omega_{2}^{\prime}, z\right) h_{2 a}^{*}\left(\bar{\omega}_{1}, \omega_{2}^{\prime}, z\right)=\delta\left(\omega_{1}-\bar{\omega}_{1}\right) \\
& \int d \omega_{2}^{\prime} h_{1 b}\left(\omega_{2}, \omega_{2}^{\prime}, z\right) h_{1 b}^{*}\left(\bar{\omega}_{2}, \omega_{2}^{\prime}, z\right) \\
& -\int d \omega_{1}^{\prime} h_{2 b}\left(\omega_{1}^{\prime}, \omega_{2}, z\right) h_{2 b}^{*}\left(\omega_{1}^{\prime}, \bar{\omega}_{2}, z\right)=\delta\left(\omega_{2}-\bar{\omega}_{2}\right) \\
& \quad d \omega_{1}^{\prime} h_{1 a}\left(\omega_{1}, \omega_{1}^{\prime}, z\right) h_{2 b}\left(\omega_{1}^{\prime}, \bar{\omega}_{2}, z\right) \\
& -\int d \omega_{2}^{\prime} h_{1 b}\left(\bar{\omega}_{2}, \omega_{2}^{\prime}, z\right) h_{2 a}\left(\omega_{1}, \omega_{2}^{\prime}, z\right)=0
\end{aligned}
$$

These relations guarantee the commutation relations $\left[\hat{a}^{(o)}\left(\omega_{1}\right), \hat{a}^{(o) \dagger}\left(\bar{\omega}_{1}\right)\right]=\delta\left(\omega_{1}-\bar{\omega}_{1}\right)$ and $\left[\hat{b}^{(o)}\left(\omega_{2}\right)\right.$, $\left.\hat{b}^{(o)^{\dagger}}\left(\bar{\omega}_{2}\right)\right]=\delta\left(\omega_{2}-\bar{\omega}_{2}\right), \quad\left[\hat{a}^{(o)}\left(\omega_{1}\right), \hat{b}^{(o)}\left(\bar{\omega}_{2}\right)\right]=0 \quad$ from Eq. (23).

\section{EIGENMODES OF HIGH GAIN PARAMETRIC PROCESSES}

Although $h$ functions have a very complicated form, we can in general use singular value decomposition method to decompose them as

$$
\begin{aligned}
& h_{1 a}\left(\omega, \omega^{\prime}\right)=\sum_{k} r_{1 a}^{(k)} \psi_{1 a}^{(k)}(\omega) \phi_{1 a}^{(k)}\left(\omega^{\prime}\right), \\
& h_{2 a}\left(\omega, \omega^{\prime}\right)=\sum_{k} r_{2 a}^{(k)} \psi_{2 a}^{(k)}(\omega) \phi_{2 a}^{(k)}\left(\omega^{\prime}\right), \\
& h_{1 b}\left(\omega, \omega^{\prime}\right)=\sum_{k} r_{1 b}^{(k)} \psi_{1 b}^{(k)}(\omega) \phi_{1 b}^{(k)}\left(\omega^{\prime}\right), \\
& h_{2 b}\left(\omega, \omega^{\prime}\right)=\sum_{k} r_{2 b}^{(k)} \psi_{2 b}^{(k)}(\omega) \phi_{2 b}^{(k)}\left(\omega^{\prime}\right) .
\end{aligned}
$$

Because of the relations (38), it can be shown (see the Appendix) that $\psi_{1 a}^{(k)}(\omega)=\psi_{2 a}^{(k)}(\omega) \equiv \psi_{k}^{(a)}(\omega), \quad \psi_{1 b}^{(k)}(\omega)=$ $\psi_{2 b}^{(k)}(\omega) \equiv \psi_{k}^{(b)}(\omega), \phi_{1 a}^{(k)}\left(\omega^{\prime}\right)=\phi_{2 b}^{(k) *}\left(\omega^{\prime}\right) \equiv \phi_{k}^{(a)}\left(\omega^{\prime}\right), \phi_{1 b}^{(k)}\left(\omega^{\prime}\right)$ $=\phi_{2 a}^{(k) *}\left(\omega^{\prime}\right) \equiv \phi_{k}^{(b)}\left(\omega^{\prime}\right), \quad r_{1 a}^{(k)}=r_{1 b}^{(k)} \equiv \cosh r_{k} G$, and $r_{2 a}^{(k)}=$ $r_{2 b}^{(k)}=\sqrt{r_{1 a}^{(k) 2}-1}=\sinh r_{k} G$. Here $\psi_{k}^{(a)}(\omega), \quad \phi_{k}^{(a)}(\omega)$, $\psi_{k}^{(b)}(\omega)$, and $\phi_{k}^{(b)}(\omega)$ are four sets of orthonormal mode functions satisfying $\int d \omega \psi_{k}^{(a, b) *}(\omega) \psi_{k^{\prime}}^{(a, b)}(\omega)=\delta_{k k^{\prime}}$ and $\int d \omega$ $\phi_{k}^{(a, b) *}(\omega) \phi_{k^{\prime}}^{(a, b)}(\omega)=\delta_{k k^{\prime}}$ and $r_{k}$ are normalized mode coefficients satisfying $\sum_{k} r_{k}^{2}=1$ with $G$ being some parameter depending on $\chi$. So $h$ functions are in the form

$$
\begin{aligned}
& h_{1 a}\left(\omega_{1}, \omega_{1}^{\prime}, z\right)=\sum_{k} \cosh \left(r_{k} G\right) \psi_{k}^{(a)}\left(\omega_{1}\right) \phi_{k}^{(a)}\left(\omega_{1}^{\prime}\right), \\
& h_{2 a}\left(\omega_{1}, \omega_{2}^{\prime}, z\right)=\sum_{k} \sinh \left(r_{k} G\right) \psi_{k}^{(a)}\left(\omega_{1}\right) \phi_{k}^{(b) *}\left(\omega_{2}^{\prime}\right), \\
& h_{1 b}\left(\omega_{2}, \omega_{2}^{\prime}, z\right)=\sum_{k} \cosh \left(r_{k} G\right) \psi_{k}^{(b)}\left(\omega_{2}\right) \phi_{k}^{(b)}\left(\omega_{2}^{\prime}\right), \\
& h_{2 b}\left(\omega_{1}^{\prime}, \omega_{2}, z\right)=\sum_{k} \sinh \left(r_{k} G\right) \psi_{k}^{(b)}\left(\omega_{1}^{\prime}\right) \phi_{k}^{(a) *}\left(\omega_{2}\right) .
\end{aligned}
$$

With these relations and orthonormal relations for $\psi_{k}^{(a, b)}(\omega)$, Eqs. (23) and (23) can be recast as

$$
\begin{aligned}
& \hat{A}_{k}^{(o)}=\cosh \left(r_{k} G\right) \hat{A}_{k}+\sinh \left(r_{k} G\right) \hat{B}_{k}^{\dagger}, \\
& \hat{B}_{k}^{(o)}=\cosh \left(r_{k} G\right) \hat{B}_{k}+\sinh \left(r_{k} G\right) \hat{A}_{k}^{\dagger},
\end{aligned}
$$

where $\quad \hat{A}_{k}^{(o)} \equiv \int d \omega \psi_{k}^{(a) *} \hat{a}^{(o)}(\omega), \quad \hat{B}_{k}^{(o)} \equiv \int d \omega \psi_{k}^{(b) *} \hat{b}^{(o)}(\omega)$, $\hat{A}_{k} \equiv \int d \omega \phi_{k}^{(a)} \hat{a}(\omega)$, and $\hat{B}_{k} \equiv \int d \omega \phi_{k}^{(b)} \hat{b}(\omega)$ define the annihilation operators for the corresponding output and input temporal modes, similar to those given in Eq. (26). Because of the orthonormal relations, they satisfy the boson commutation relation for annihilation operators.

Because of the $\delta$ function in the initial conditions (37), we cannot solve directly the differential-integral equations (35) and (36). In order to proceed, let us write $\bar{h}_{1 a}\left(\omega_{1}, \omega_{1}^{\prime}, z\right) \equiv$ $h_{1 a}\left(\omega_{1}, \omega_{1}^{\prime}, z\right)-\delta\left(\omega_{1}-\omega_{1}^{\prime}\right) \quad$ and $\quad \bar{h}_{1 b}\left(\omega_{1}, \omega_{1}^{\prime}, z\right) \equiv h_{1 b}\left(\omega_{1}\right.$, $\left.\omega_{1}^{\prime}, z\right)-\delta\left(\omega_{1}-\omega_{1}^{\prime}\right)$. Furthermore, for a specific parametric process from four-wave mixing in optical fiber with a Gaussian pumping profile, we have $A_{p}\left(\omega_{3}\right)=$ $A_{0}^{2} \exp \left[-\left(\omega_{3}-2 \omega_{p 0}\right)^{2} / 4 \sigma_{p}^{2}\right]$ with a pump bandwidth of $\sigma_{p}$ and pump amplitude $A_{0}$ [10]. We can introduce some new dimensionless variables, $\zeta \equiv z / L_{0}$ and $\Omega_{j} \equiv\left(\omega_{j}-\omega_{j 0}\right) / \sigma_{p}$ $(j=1,2)$, with $L_{0}$ the length of the nonlinear medium and $\omega_{j 0}(j=1,2, p)$ the central frequency of the corresponding 
fields. Then we can make Eqs. (35) and (36) dimensionless as

$$
\begin{aligned}
& \frac{d}{d \zeta} \bar{h}_{1 a}\left(\Omega_{1}, \Omega_{1}^{\prime}, \zeta\right)=\int d \Omega_{2} f\left(\Omega_{1}, \Omega_{2}, \zeta\right) h_{2 b}^{*}\left(\Omega_{1}^{\prime}, \Omega_{2}, \zeta\right), \\
& \frac{d}{d \zeta} h_{2 b}\left(\Omega_{1}^{\prime}, \Omega_{2}, \zeta\right) \\
& \quad=f\left(\Omega_{1}^{\prime}, \Omega_{2}, \zeta\right)+\int d \Omega_{1} f\left(\Omega_{1}, \Omega_{2}, \zeta\right) \bar{h}_{1 a}^{*}\left(\Omega_{1}, \Omega_{1}^{\prime}, \zeta\right)
\end{aligned}
$$

and

$$
\begin{aligned}
& \frac{d}{d \zeta} \bar{h}_{1 b}\left(\Omega_{2}, \Omega_{2}^{\prime}, \zeta\right)=\int d \Omega_{1} f\left(\Omega_{1}, \Omega_{2}, \zeta\right) h_{2 a}^{*}\left(\Omega_{1}, \Omega_{2}^{\prime}, \zeta\right), \\
& \frac{d}{d \zeta} h_{2 a}\left(\Omega_{1}, \Omega_{2}^{\prime}, \zeta\right) \\
& \quad=f\left(\Omega_{1}, \Omega_{2}^{\prime}, \zeta\right)+\int d \Omega_{2} f\left(\Omega_{1}, \Omega_{2}, \zeta\right) \bar{h}_{1 b}^{*}\left(\Omega_{2}, \Omega_{2}^{\prime}, \zeta\right),
\end{aligned}
$$

where $f\left(\Omega_{1}, \Omega_{2}, \zeta\right) \equiv K e^{-i \zeta \Delta k L_{0}} \exp \left[-\left(\Omega_{1}+\Omega_{2}\right)^{2} / 4\right]$, with $K \equiv 2 \pi L_{0} A_{0}^{2} \sigma_{p} \chi$ the dimensionless pump parameter and $h\left(\Omega_{1}, \Omega_{2}\right)$ functions dimensionless and related to $h(\omega)$ functions by $h\left(\Omega_{1}, \Omega_{2}\right) \equiv \sigma_{p} h\left(\omega_{1}, \omega_{2}\right)$. Phase mismatch $\Delta k L_{0}$ can be adjusted according to the dispersion of the nonlinear medium and in general has a linear form of $\Delta k L_{0}=\Omega_{1} / \Delta_{1}+$ $\Omega_{2} / \Delta_{2}$ with the parameters $\Delta_{1}$ and $\Delta_{2}$ determined by medium dispersion, pump bandwidth $\sigma_{p}$, and medium length $L_{0}$. The initial conditions (37) change to

$$
\begin{aligned}
& \bar{h}_{1 a}\left(\Omega_{1}, \Omega_{1}^{\prime}, \zeta=0\right)=0, \\
& \bar{h}_{1 b}\left(\Omega_{2}, \Omega_{2}^{\prime}, \zeta=0\right)=0, \\
& h_{2 a}\left(\Omega_{1}, \Omega_{2}^{\prime}, \zeta=0\right)=0, \\
& h_{2 b}\left(\Omega_{1}^{\prime}, \Omega_{2}, \zeta=0\right)=0 .
\end{aligned}
$$

Note that when the pump parameter is small $K \ll 1$, $h_{2 a}\left(\Omega_{1}, \Omega_{2}\right)$ has an approximate analytical solution

$$
\begin{aligned}
h_{2 a}\left(\Omega_{1}, \Omega_{2}, \zeta=1\right) \approx & K e^{-i \Delta k L_{0} / 2} \operatorname{sinc}\left(\frac{\Delta k L_{0}}{2}\right) \\
& \times \exp \left(-\frac{\left(\Omega_{1}+\Omega_{2}\right)^{2}}{4}\right),
\end{aligned}
$$

which is exactly the joint spectral function $\Phi\left(\omega_{1}, \omega_{2}\right)$ in Eq. (22) after changing to dimensionless quantities. However, for a sizable $K$, we cannot solve the differential-integral equations (42) and (43) analytically. Next we will solve them numerically, subject to initial conditions (44).

\section{NUMERICAL SOLUTIONS}

Let us use a nonlinear fiber as the nonlinear medium. We use parameters similar to those given in Ref. [33] for a real piece of 300-m-long dispersion-shifted nonlinear fiber. We obtain the dimensionless parameters $1 / \Delta_{1}=0.785$ and $1 / \Delta_{2}=-0.471$. The numerical solution of the amplitude of $h_{2 a}\left(\Omega_{1}, \Omega_{2}\right)$ is shown in Fig. 4 for four values of the pump parameter $K: K=0.01,2,4,10$. For small $K(\ll 1)$ [Fig. 4(a)], $h_{2 a}\left(\Omega_{1}, \Omega_{2}\right)$ is exactly the joint spectral function $\Phi\left(\omega_{1}, \omega_{2}\right)$

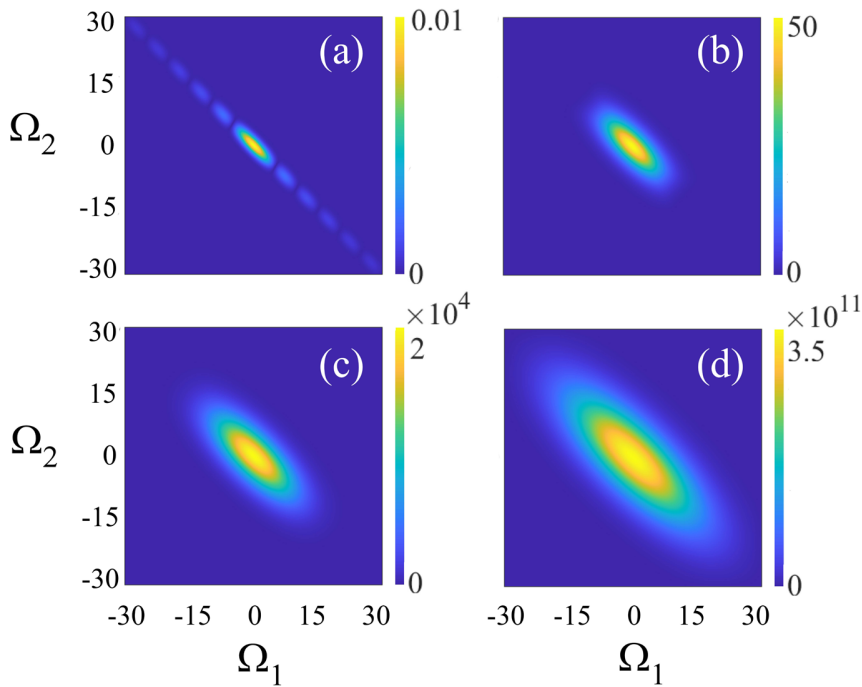

FIG. 4. Contour plot of the amplitude of $h_{2 a}\left(\Omega_{1}, \Omega_{2}\right)$ for (a) $K=$ 0.01 , (b) $K=2$, (c) $K=4$, and (d) $K=10$, with $\Omega_{i} \equiv\left(\omega_{i}-\right.$ $\left.\omega_{i 0}\right) / \sigma_{p}(i=1,2)$.

given in Eq. (22), similar to Fig. 2(a) of Ref. [33]. The shape starts to broaden as $K$ increases. This can be seen in the profile change of the eigenfunction $\psi_{1}^{(a)}\left(\Omega_{1}\right)$ obtained from singular value decomposition when we plot it in Fig. 5 for four values of $K(0.01,2,4$, and 10). To show the trend, we plot the full width at half maximum of $\left|\psi_{1}^{(a)}\left(\Omega_{1}\right)\right|$ as a function of $K$ in the inset of Fig. 5.

The change of the transfer function $h_{2 a}\left(\Omega_{1}, \Omega_{2}\right)$ with the pump parameter $K$ will lead to mode structure change. This is reflected in the change of the distribution of the mode coefficients $\left\{r_{k}\right\}(k=2-8)$, whose normalized values to $r_{1}$ are plotted in Fig. 6 as a function of the pump parameter $K[\mathrm{M} k$ denotes mode $k(k=2-8)$ ]. The trend shows the increasing weight of the higher-order modes in addition to the broadening of the mode functions as $K$ increases.

The multimode nature and the broadening of the mode functions with pump parameter $K$ are due to the values of $\Delta_{1}$ and $\Delta_{2}$ in $\Delta k L_{0}$ for a realistic nonlinear fiber case, which

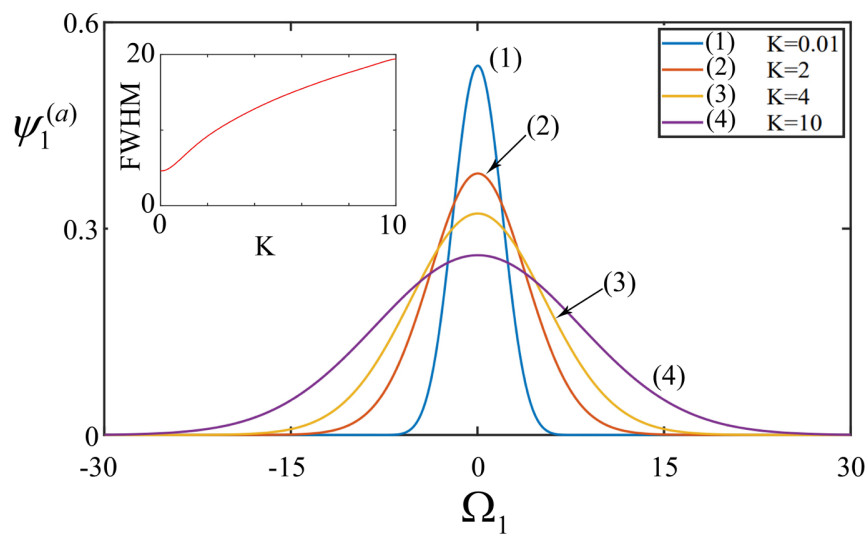

FIG. 5. Amplitude function $\psi_{1}^{(a)}$ of mode 1 for pump parameter $K=0.01,2,4,10$, showing the broadening of the width. The inset shows the full width at half maximum (FWHM) of the mode 1 function as a function of the pump parameter $K$. 


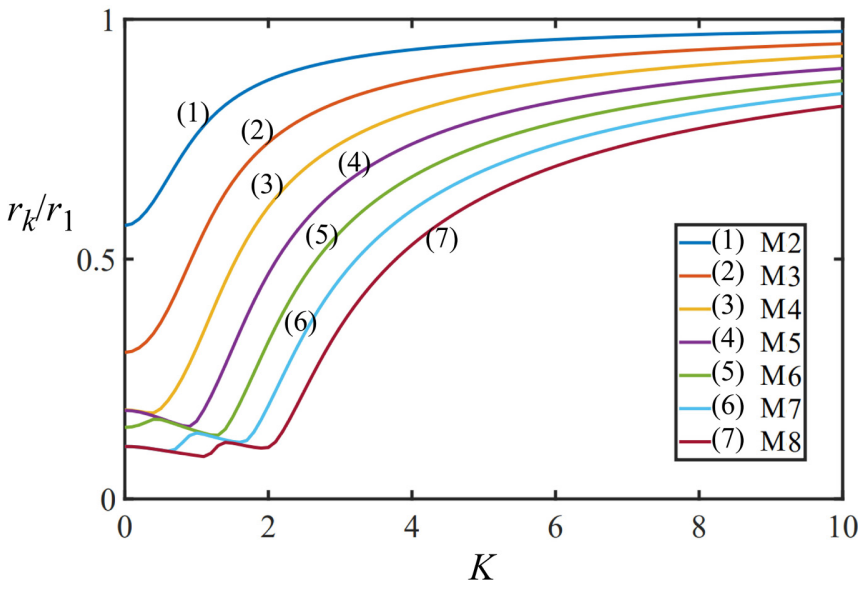

FIG. 6. Mode coefficients $r_{k} / r_{1}$ as a function of dimensionless pump parameter $K$. Here $\mathrm{M} k$ denotes mode $k(k=2-8)$.

gives rise to an asymmetric sinc function in $\Phi$ of Eq. (22) or $h_{2 a}\left(\Omega_{1}, \Omega_{2}\right)$ at small $K$ [Fig. 4(a)]. In principle, we can adjust the dispersion parameters of the fiber to change $\Delta_{1}$ and $\Delta_{2}$ in $\Delta k L_{0}$ [34]. It is found that the initial $h_{2 a}\left(\Omega_{1}, \Omega_{2}\right)$ when $K \ll 1$ is nearly round or factorized if we can have parameters $1 / \Delta_{1}=2.198$ and $1 / \Delta_{2}=-2.198$. This situation is shown in $h_{2 a}\left(\Omega_{1}, \Omega_{2}\right)$ in Fig. 7(a). The initial $r_{k}$ distribution is indeed close to single mode with high-order $r_{k}$ much smaller than 1 . This can be seen in Fig. 8 for $K=0$. However, the trend of mode spreading for large $K$ in Fig. 6 persists in Fig. 8.

On the other hand, a close look at Figs. 7(c) and 7(d) for large $K$ shows that the function $h_{2 a}$ is still pretty round or nearly single mode. In fact, we find from mode decomposition in Eqs. (40) that the coefficient of each mode is $\sinh ^{2} r_{k} G$, which becomes $0.25 e^{2 r_{k} G}$ for large $r_{k} G$. So the ratio of coefficients of the first mode to higher mode is then $e^{2\left(r_{1}-r_{k}\right) G} \gg 1$ for large $G$, that is, the first mode will dominate in the mode
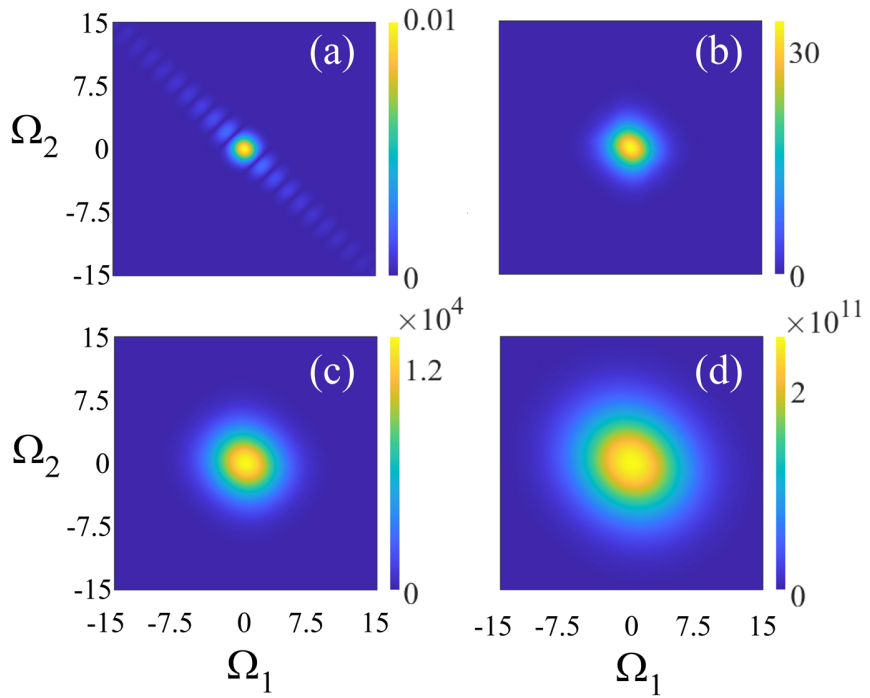

FIG. 7. Contour plot of the amplitude of $h_{2 a}\left(\Omega_{1}, \Omega_{2}\right)$ for the case of $1 / \Delta_{1}=2.198$ and $1 / \Delta_{2}=-2.198$ for (a) $K=0.01$, (b) $K=2$, (c) $K=4$, and (d) $K=10$, with $\Omega_{i} \equiv\left(\omega_{i}-\omega_{i 0}\right) / \sigma_{p}(i=1,2)$.

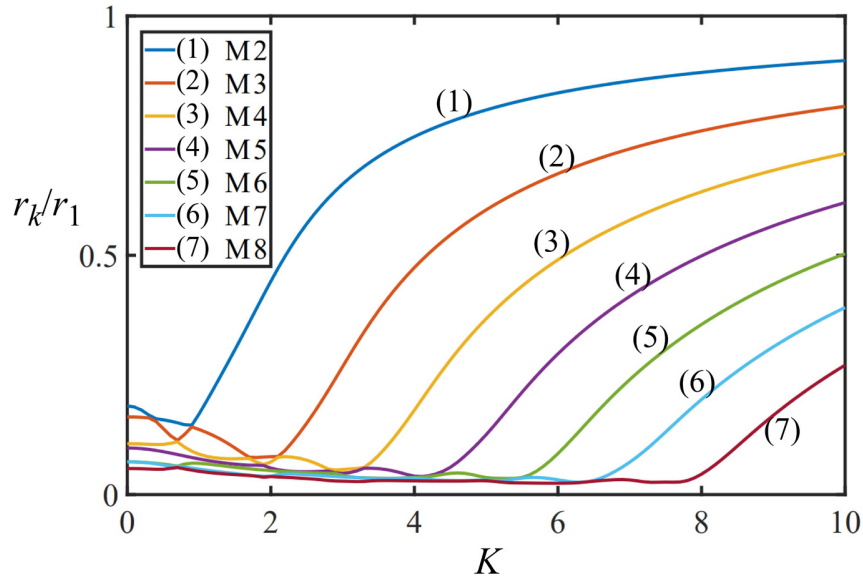

FIG. 8. Mode coefficients $r_{k} / r_{1}$ as a function of dimensionless pump parameter $K$ for the nearly factorized case of $1 / \Delta_{1}=2.198$ and $1 / \Delta_{2}=-2.198$. Here $\mathrm{M} k$ denotes mode $k(k=2-8)$.

decomposition in Eqs. (40) at large $K$ (or $G$ ) [35], which leads to a nearly round (or factorized) $h_{2 a}$.

Furthermore, we can look at the mode purity of the output state. It is known that if we discard the other field, one of the output fields from a parametric amplifier is in a thermal state. For broadband pulsed pumping, it becomes a multimode thermal field. Mode purity for a pulsed multimode thermal field was studied in Ref. [36] with the temporal mode format described here. It was found that mode purity can be characterized by the normalized intensity correlation function $g^{(2)}$ defined as

$$
g^{(2)} \equiv \frac{\left\langle I^{2}\right\rangle}{\langle I\rangle^{2}} \equiv 1+\frac{1}{M}=1+\frac{\sum_{k} I_{k}^{2}}{\left(\sum_{k} I_{k}\right)^{2}},
$$

where the quantity $M$ gives the average mode number of the field [37] ( $M=1$ gives the pure single-mode case and $M=N$ for $N$ identical $\left.I_{k}=I_{0}, k=1,2, \ldots, N\right)$ and $I_{k}$ is the intensity of mode $k$. From Eq. (41) we find that $I_{k}=\sinh ^{2} r_{k} G$ for vacuum input to the parametric amplifier. So Eq. (46) gives

$$
M=\frac{\left(\sum_{k} \sinh ^{2} r_{k} G\right)^{2}}{\sum_{k} \sinh ^{4} r_{k} G} .
$$

Obviously, for the single-mode case with $r_{1}=1$ and $r_{k}=0$ $(k>1)$, we have $M=1$. Equation (47) was first derived by Christ et al. [15] for temporal modes and by Sharapova et al. [38] and Dyakonov et al. [39] for spatial modes in high gain parametric processes. For the two cases shown in Figs. 4 and 7 , we evaluate the average mode number $M$ in Eq. (47) as a function of pump parameter $K$ and plot the results in Fig. 9. It can be seen that the average mode number $M$ indeed drops first as $K$ increases. This drop is consistent with the dominance of the first mode as $K$ increases. However, the drop stops at around $K=2$ when a minimum of $M$ is reached; $M$ starts to slowly increase after $K>2$. This is due to the increase of $r_{k}$ for higher-order modes as $K$ increases, as shown in Figs. 6 and 8. The minimum $M$ value depends on the initial $M$ at low $K(\ll 1)$. Thus, it is better to have a nearly single-mode situation at low pump power. 


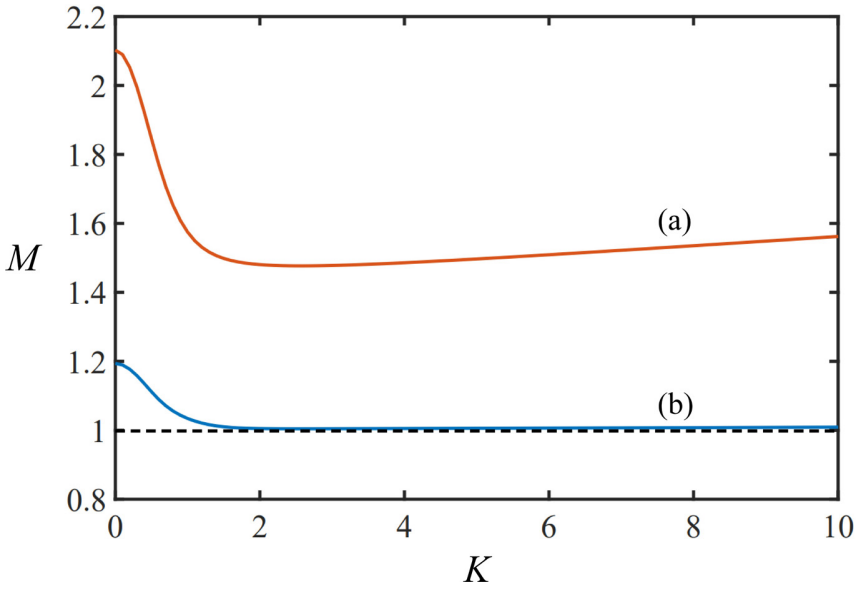

FIG. 9. Average mode number $M$ as a function of pump parameter $K$ for the cases shown in Fig. 4 [orange, curve (a)] and Fig. 7 [blue, curve (b)]. The dashed line corresponds to $M=1$.

\section{SUMMARY AND DISCUSSION}

We have studied the mode structure for a broadband parametric amplifier at different gains by using an input-output approach that avoids the crucial issue of time ordering in the Hamiltonian. Contrary to previous studies where the timeordering issue was not treated, the mode structure changes as the gain increases in the sense that both the mode distribution and the mode functions broaden with the increase of the gain. Although the mode number, a quantity that characterizes the total number of modes, drops initially as the gain changes from low to high due to the dominance of the first mode, it reaches a minimum value before slowly increasing due to the broadening of the mode distribution.

The mode structure change with the gain will have a profound impact on the application of broadband parametric processes in quantum technology with continuous variables, which relies on the homodyne detection method as the domi- nant measurement technique. Even though the output of the broadband parametric processes is of a multimode nature, because of the orthogonality of the modes in the processes, we can choose the local oscillators of the homodyne measurement to match the mode functions of the output and select specific modes for study [18]. The change of mode structure and functions with the gain means that we will need to measure them constantly at different gains as we change the operation condition. Fortunately, methods have been developed recently for the direct measurement of mode functions and mode coefficients of parametric processes [18,40].

\section{ACKNOWLEDGMENTS}

We would like to thank Prof. M. Raymer for helpful discussion. This work was supported by U.S. National Science Foundation (Grant No. 1806425).

\section{APPENDIX} write

In general, we can use singular value decomposition to

$$
\begin{aligned}
& h_{1 a}\left(\omega, \omega^{\prime}\right)=\sum_{k} r_{1 a}^{(k)} \psi_{1 a}^{(k)}(\omega) \phi_{1 a}^{(k)}\left(\omega^{\prime}\right), \\
& h_{2 a}\left(\omega, \omega^{\prime}\right)=\sum_{k} r_{2 a}^{(k)} \psi_{2 a}^{(k)}(\omega) \phi_{2 a}^{(k)}\left(\omega^{\prime}\right), \\
& h_{1 b}\left(\omega, \omega^{\prime}\right)=\sum_{k} r_{1 b}^{(k)} \psi_{1 b}^{(k)}(\omega) \phi_{1 b}^{(k)}\left(\omega^{\prime}\right), \\
& h_{2 b}\left(\omega, \omega^{\prime}\right)=\sum_{k} r_{2 b}^{(k)} \psi_{2 b}^{(k)}(\omega) \phi_{2 b}^{(k)}\left(\omega^{\prime}\right),
\end{aligned}
$$

where $\psi_{1 a}^{(k)}(\omega), \phi_{1 a}^{(k)}\left(\omega^{\prime}\right), \ldots$ are eight sets of orthonormal mode functions satisfying $\int d \omega \psi_{1 a}^{(k)}(\omega) \psi_{1 a}^{\left(k^{\prime}\right) *}(\omega)=\delta_{k, k^{\prime}}, \ldots$ Using the decomposition in Eq. (A1) and orthonormal relations, we write the left-hand side of Eq. (38a) as

$$
\begin{aligned}
& \int d \omega^{\prime \prime} h_{1 a}\left(\omega, \omega^{\prime \prime}\right) h_{1 a}^{*}\left(\omega^{\prime}, \omega^{\prime \prime}\right)-\int d \omega^{\prime \prime} h_{2 a}\left(\omega, \omega^{\prime \prime}\right) h_{2 a}^{*}\left(\omega^{\prime}, \omega^{\prime \prime}\right) \\
& =\int d \omega^{\prime \prime} \sum_{k, k^{\prime}} r_{1 a}^{(k)} r_{1 a}^{\left(k^{\prime}\right)} \psi_{1 a}^{(k)}(\omega) \psi_{1 a}^{\left(k^{\prime}\right) *}\left(\omega^{\prime}\right) \phi_{1 a}^{(k)}\left(\omega^{\prime \prime}\right) \phi_{1 a}^{\left(k^{\prime}\right) *}\left(\omega^{\prime \prime}\right)-\int d \omega^{\prime \prime} \sum_{k, k^{\prime}} r_{2 a}^{(k)} r_{2 a}^{\left(k^{\prime}\right)} \psi_{2 a}^{(k)}(\omega) \psi_{2 a}^{\left(k^{\prime}\right) *}\left(\omega^{\prime}\right) \phi_{2 a}^{(k)}\left(\omega^{\prime \prime}\right) \phi_{2 a}^{\left(k^{\prime}\right) *}\left(\omega^{\prime \prime}\right) \\
& =\sum_{k, k^{\prime}} r_{1 a}^{(k)} r_{1 a}^{\left(k^{\prime}\right)} \psi_{1 a}^{(k)}(\omega) \psi_{1 a}^{\left(k^{\prime}\right) *}\left(\omega^{\prime}\right) \delta_{k, k^{\prime}}-\sum_{k, k^{\prime}} r_{2 a}^{(k)} r_{2 a}^{\left(k^{\prime}\right)} \psi_{2 a}^{(k)}(\omega) \psi_{2 a}^{\left(k^{\prime}\right) *}\left(\omega^{\prime}\right) \delta_{k, k^{\prime}} \\
& =\sum_{k} r_{1 a}^{(k) 2} \psi_{1 a}^{(k)}(\omega) \psi_{1 a}^{(k) *}\left(\omega^{\prime}\right)-\sum_{k} r_{2 a}^{(k) 2} \psi_{2 a}^{(k)}(\omega) \psi_{2 a}^{(k) *}\left(\omega^{\prime}\right) .
\end{aligned}
$$

Using the completeness of the mode function $\left\{\psi_{1 a}^{(k)}(\omega)\right\}, \sum_{k} \psi_{1 a}^{(k)}(\omega) \psi_{1 a}^{(k) *}\left(\omega^{\prime}\right)=\delta\left(\omega-\omega^{\prime}\right)$, we can rewrite Eq. (38a) as

$$
\sum_{k} r_{2 a}^{(k) 2} \psi_{2 a}^{(k)}(\omega) \psi_{2 a}^{(k) *}\left(\omega^{\prime}\right)=\sum_{k}\left(r_{1 a}^{(k) 2}-1\right) \psi_{1 a}^{(k)}(\omega) \psi_{1 a}^{(k) *}\left(\omega^{\prime}\right)
$$

Treating $k$ as the row index and $\omega$ as the column index of a matrix, we can consider the mode function $\psi^{(k)}(\omega)$ as the matrix element of $\Psi$ with $\{\Psi\}_{k, \omega} \equiv \psi^{(k)}(\omega)$ (we have dropped subscripts $1 a$ and $2 a$ for clarity). Then Eq. (A3) is equivalent to the 
following matrix equation:

$$
\left[\Psi_{2 a}\right]^{\dagger}\left(\begin{array}{ccc}
r_{2 a}^{(1) 2} & 0 & \ldots \\
0 & r_{2 a}^{(2) 2} & \ldots \\
\vdots & \vdots & \ddots
\end{array}\right) \Psi_{2 a}=\left[\Psi_{1 a}\right]^{\dagger}\left(\begin{array}{ccc}
r_{1 a}^{(1) 2}-1 & 0 & \ldots \\
0 & r_{1 a}^{(2) 2}-1 & \ldots \\
\vdots & \vdots & \ddots
\end{array}\right) \Psi_{1 a} .
$$

Multiplying the left-hand side of (A4) by the matrix $\Psi_{1 a}$ and the right-hand side by $\left[\Psi_{1 a}\right]^{\dagger}$ and using the orthonormal relation $\int d \omega \psi_{1 a}^{(k)}(\omega) \psi_{1 a}^{\left(k^{\prime}\right) *}(\omega)=\delta_{k, k^{\prime}}$, we obtain a rotational transformation

$$
\mathrm{R}\left(\begin{array}{ccc}
r_{2 a}^{(1) 2} & 0 & \cdots \\
0 & r_{2 a}^{(2) 2} & \cdots \\
\vdots & \vdots & \ddots
\end{array}\right) \mathrm{R}^{\dagger}=\left(\begin{array}{ccc}
r_{1 a}^{(1) 2}-1 & 0 & \cdots \\
0 & r_{1 a}^{(2) 2}-1 & \cdots \\
\vdots & \vdots & \ddots
\end{array}\right)
$$

with transformation matrix $R \equiv \Psi_{1 a}\left[\Psi_{2 a}\right]^{\dagger}$. The only solution for the above is $\mathrm{R}=\mathrm{I}$, or $\int d \omega \psi_{1 a}^{(k)}(\omega) \psi_{2 a}^{\left(k^{\prime}\right) *}(\omega)=\delta_{k, k^{\prime}}$. With the uniqueness of the mode function set $\left\{\psi_{1 a}^{(k)}(\omega)\right\}$, we have $\psi_{1 a}^{(k)}(\omega)=\psi_{2 a}^{(k)}(\omega)$ and then $r_{1 a}^{(k) 2}-r_{2 a}^{(k) 2}=1$. Similarly, using Eq. (38b), we have $\psi_{1 b}^{(k)}(\omega)=\psi_{2 b}^{(k)}(\omega)$ and $r_{1 b}^{(k) 2}-r_{2 b}^{(k) 2}=1$.

Next let us use Eq. (38c) and rewrite it as

$$
\begin{aligned}
& \int d \omega^{\prime \prime} \sum_{k, k^{\prime}} r_{1 a}^{(k)} r_{2 b}^{\left(k^{\prime}\right)} \psi_{1 a}^{(k)}(\omega) \psi_{1 b}^{\left(k^{\prime}\right)}\left(\omega^{\prime}\right) \phi_{1 a}^{(k)}\left(\omega^{\prime \prime}\right) \phi_{2 b}^{\left(k^{\prime}\right)}\left(\omega^{\prime \prime}\right) \\
& -\int d \omega^{\prime \prime} \sum_{k, k^{\prime}} r_{2 a}^{(k)} r_{1 b}^{\left(k^{\prime}\right)} \psi_{1 a}^{(k)}(\omega) \psi_{1 b}^{\left(k^{\prime}\right)}\left(\omega^{\prime}\right) \\
& \times \phi_{2 a}^{(k)}\left(\omega^{\prime \prime}\right) \phi_{1 b}^{\left(k^{\prime}\right)}\left(\omega^{\prime \prime}\right)=0 .
\end{aligned}
$$

Multiplying both sides by $\psi_{1 a}^{\left(k_{1}\right) *}(\omega) \psi_{1 b}^{\left(k_{2}\right) *}\left(\omega^{\prime}\right)$ and integrating $\omega$ and $\omega^{\prime}$, with orthonormal relations for $\psi_{1 a}^{(k)}(\omega)$ and $\psi_{1 b}^{\left(k^{\prime}\right)}\left(\omega^{\prime}\right)$, we obtain

$$
\cosh r_{a}^{\left(k_{1}\right)} \sinh r_{b}^{\left(k_{2}\right)} R_{k_{2}, k_{1}}=\sinh r_{a}^{\left(k_{1}\right)} \cosh r_{b}^{\left(k_{2}\right)} R_{k_{2}, k_{1}}^{\prime},
$$

where we set $r_{1 a}^{(k)} \equiv \cosh r_{a}^{(k)}$ and $r_{1 b}^{(k)} \equiv \cosh r_{b}^{(k)}$ and then $r_{2 a}^{(k)}=\sqrt{r_{1 a}^{(k) 2}-1}=\sinh r_{a}^{(k)}$ and $r_{2 b}^{(k)}=\sqrt{r_{1 b}^{(k) 2}-1}=$ $\sinh r_{b}^{(k)}$; in addition, $R_{k_{2}, k_{1}}^{\prime} \equiv \int d \omega \phi_{1 b}^{\left(k_{2}\right)}(\omega) \phi_{2 a}^{\left(k_{1}\right)}(\omega)$ and $R_{k_{2}, k_{1}} \equiv \int d \omega \phi_{2 b}^{\left(k_{2}\right)}(\omega) \phi_{1 a}^{\left(k_{1}\right)}(\omega)$. Switching back to the notation $k=k_{1}$ and $k^{\prime}=k_{2}$, we have

$$
\begin{aligned}
& R_{k^{\prime}, k}=\tanh r_{a}^{(k)} \operatorname{coth} r_{b}^{\left(k^{\prime}\right)} R_{k^{\prime}, k}^{\prime}, \\
& R_{k^{\prime}, k}^{\prime}=\operatorname{coth} r_{a}^{(k)} \tanh r_{b}^{\left(k^{\prime}\right)} R_{k^{\prime}, k},
\end{aligned}
$$

which, in matrix form, is simply $\mathrm{R}=\mathrm{C}_{b} \mathrm{R}^{\prime} \mathrm{C}_{a}^{-1}$ or $\mathrm{R}^{\prime}=$ $\mathrm{C}_{b}^{-1} \mathrm{RC}_{a}$, with

$$
\mathrm{C}_{a} \equiv\left(\begin{array}{ccc}
\operatorname{coth} r_{a}^{(1)} & 0 & \cdots \\
0 & \operatorname{coth} r_{a}^{(2)} & \cdots \\
\vdots & \vdots & \ddots
\end{array}\right)
$$

and

$$
\mathrm{C}_{b} \equiv\left(\begin{array}{ccc}
\operatorname{coth} r_{b}^{(1)} & 0 & \cdots \\
0 & \operatorname{coth} r_{b}^{(2)} & \cdots \\
\vdots & \vdots & \ddots
\end{array}\right)
$$

Now notice that $R R^{\dagger}=I=R^{\prime} R^{\prime \dagger}$, or using the matrix form of Eqs. (A8), we obtain

$$
\mathrm{C}_{b} \mathrm{R}^{\prime} \mathrm{C}_{a}^{-1} \mathrm{C}_{a}^{-1} \mathrm{R}^{\prime \dagger} \mathrm{C}_{b}=\mathrm{I}, \quad \mathrm{C}_{b}^{-1} \mathrm{RC}_{a} \mathrm{C}_{a} \mathrm{R}^{\dagger} \mathrm{C}_{b}^{-1}=\mathrm{I}
$$

Rewriting (A11), we have

$$
\mathrm{R}^{\prime} \mathrm{C}_{a}^{-2} \mathrm{R}^{\prime \dagger}=\mathrm{C}_{b}^{-2}, \quad \mathrm{RC}_{a}^{2} \mathrm{R}^{\dagger}=\mathrm{C}_{b}^{2} .
$$

Since matrices $\mathrm{C}_{a}$ and $\mathrm{C}_{b}$ are both diagonalized, the above expressions are true only if $\mathrm{R}^{\prime}=\mathrm{I}=\mathrm{R}$ ( or $\phi_{1 a}^{(k)}=\phi_{2 b}^{(k) *}$ and $\left.\phi_{1 b}^{(k)}=\phi_{2 a}^{(k) *}\right)$ and $\mathrm{C}_{a}=\mathrm{C}_{b}\left(\right.$ or $\left.r_{a}^{(k)}=r_{b}^{(k)}\right)$. Setting $r_{a}^{(k)}=$ $r_{b}^{(k)} \equiv r_{k} G$ with $r_{k}$ normalized, $\sum_{k} r_{k}^{2}=1$, and $\phi_{1 a}^{(k)}=\phi_{2 b}^{(k) *} \equiv$ $\phi_{k}^{(a)}, \phi_{1 b}^{(k)}=\phi_{2 a}^{(k) *} \equiv \phi_{k}^{(b)}, \psi_{1 a}^{(k)}(\omega)=\psi_{2 a}^{(k)}(\omega) \equiv \psi_{k}^{(a)}(\omega)$, and $\psi_{1 b}^{(k)}(\omega)=\psi_{2 b}^{(k)}(\omega) \equiv \psi_{k}^{(b)}(\omega)$ in Eq. (A1), we obtain Eq. (40).
[1] D. F. Walls and G. J. Milburn, Quantum Optics, 2nd ed. (Springer, Berlin, 2008).

[2] M. D. Reid, P. D. Drummond, W. P. Bowen, E. G. Cavalcanti, P. K. Lam, H. A. Bachor, U. L. Andersen, and G. Leuchs, Colloquium: The Einstein-Podolsky-Rosen paradox: From concepts to applications, Rev. Mod. Phys. 81, 1727 (2009).

[3] X. Su, S. Hao, X. Deng, L. Ma, M. Wang, X. Jia, C. Xie, and K. Peng, Gate sequence for continuous variable one-way quantum computation, Nat. Commun. 4, 2828 (2013).

[4] M. V. Larsen, X. Guo, C. R. Breum, J. S. Neergaard-Nielsen, and U. L. Andersen, Deterministic generation of a twodimensional cluster state, Science 366, 369 (2019).

[5] W. Asavanant, Y. Shiozawa, S. Yokoyama, B. Charoensombutamon, H. Emura, R. N. Alexander, S. Takeda, J.-I. Yoshikawa, N. C. Menicucci, H. Yonezawa, and A. Furusawa, Generation of time-domain-multiplexed two-dimensional cluster state, Science 366, 373 (2019).

[6] J. Zhao, K. Liu, H. Jeng, M. Gu, J. Thompson, P. K. Lam, and S. M. Assad, A high-fidelity heralded quantum squeezing gate, Nat. Photon. 14, 306 (2020).

[7] S. Takeda, T. Mizuta, M. Fuwa, P. van Loock, and A. Furusawa, Deterministic quantum teleportation of photonic quantum bits by a hybrid technique, Nature (London) 500, 315 (2013).

[8] X. Su, C. Tian, X. Deng, Q. Li, C. Xie, and K. Peng, Quantum Entanglement Swapping between Two Multipartite Entangled States, Phys. Rev. Lett. 117, 240503 (2016).

[9] X. Guo, C. R. Breum, J. Borregaard, S. Izumi, M. V. Larsen, T. Gehring, M. Christandl, J. S. Neergaard-Nielsen, and U. L. Andersen, Distributed quantum sensing in a continuousvariable entangled network, Nat. Phys. 16, 281 (2020). 
[10] O. Aytür and P. Kumar, Pulsed Twin Beams of Light, Phys. Rev. Lett. 65, 1551 (1990).

[11] A. Eckstein, A. Christ, P. J. Mosley, and C. Silberhorn, Highly Efficient Single-Pass Source of Pulsed Single-Mode Twin Beams of Light, Phys. Rev. Lett. 106, 013603 (2011).

[12] X. Guo, N. Liu, Y. Liu, X. Li, and Z. Y. Ou, Generation of continuous variable quantum entanglement using a fiber optical parametric amplifier, Opt. Lett. 41, 653 (2016).

[13] X. Guo, X. Li, N. Liu, and Z. Y. Ou, Multimode theory of pulsed-twin-beam generation using a high-gain fiber-optical parametric amplifier, Phys. Rev. A 88, 023841 (2013).

[14] W. Wasilewski, A. I. Lvovsky, K. Banaszek, and C. Radzewicz, Pulsed squeezed light: Simultaneous squeezing of multiple modes, Phys. Rev. A 73, 063819 (2006).

[15] A. Christ, K. Laiho, A. Eckstein, K. N. Cassemiro, and C. Silberhorn, Probing multimode squeezing with correlation functions, New J. Phys. 13, 033027 (2011).

[16] C. K. Law, I. A. Walmsley, and J. H. Eberly, Continuous Frequency Entanglement: Effective Finite Hilbert Space and Entropy Control, Phys. Rev. Lett. 84, 5304 (2000).

[17] L. Cui, J. Su, J. Li, Y. Liu, X. Li, and Z. Y. Ou, Quantum state engineering by nonlinear quantum interference, Phys. Rev. A 102, 033718 (2020)

[18] N. Huo, Y. Liu, J. Li, L. Cui, X. Chen, R. Palivela, T. Xie, X. Li, and Z. Y. Ou, Direct Temporal Mode Measurement for the Characterization of Temporally Multiplexed High Dimensional Quantum Entanglement in Continuous Variables, Phys. Rev. Lett. 124, 213603 (2020).

[19] J. Chen, X. Li, and P. Kumar, Two-photon-state generation via four-wave mixing in optical fibers, Phys. Rev. A 72, 033801 (2005).

[20] N. Quesada and J. E. Sipe, Effects of time ordering in quantum nonlinear optics, Phys. Rev. A 90, 063840 (2014).

[21] P. R. Sharapova, G. Frascella, M. Riabinin, A. M. Pérez, O. V. Tikhonova, S. Lemieux, R. W. Boyd, G. Leuchs, and M. V. Chekhova, Properties of bright squeezed vacuum at increasing brightness, Phys. Rev. Res. 2, 013371 (2020).

[22] N. Quesada, G. Triginer, M. D. Vidrighin, and J. E. Sipe, Theory of high-gain twin-beam generation in waveguides: From Maxwell's equations to efficient simulation, Phys. Rev. A 102, 033519 (2020).

[23] D. V. Reddy, M. G. Raymer, C. J. McKinstrie, L. Mejling, and K. Rottwitt, Temporal mode selectivity by frequency conversion in second-order nonlinear optical waveguides, Opt. Express 21, 013840 (2013).

[24] A. Eckstein, B. Brecht, and C. Silberhorn, A quantum pulse gate based on spectrally engineered sum frequency generation, Opt. Express 19, 13770 (2011).

[25] B. Brecht, A. Eckstein, R. Ricken, V. Quiring, H. Suche, L. Sansoni, and C. Silberhorn, Demonstration of coherent time-frequency schmidt mode selection using dispersionengineered frequency conversion, Phys. Rev. A 90, 030302(R) (2014).

[26] D. V. Reddy, M. G. Raymer, and C. J. McKinstrie, Efficient sorting of quantum-optical wave packets by temporal-mode interferometry, Opt. Lett. 39, 2924 (2014).

[27] Z. Y. Ou, Enhancement of the phase-measurement sensitivity beyond the standard quantum limit by a nonlinear interferometer, Phys. Rev. A 85, 023815 (2012).

[28] Z. Y. Ou and X. Li, Quantum SU(1, 1) interferometers: Basic principles and applications, APL Photon. 5, 080902 (2020).

[29] F. Hudelist, J. Kong, C. Liu, J. Jing, Z. Y. Ou, and W. Zhang, Quantum metrology with parametric amplifier-based photon correlation interferometers, Nat. Commun. 5, 3049 (2014).

[30] G. B. Lemos, V. Borish, G. D. Cole, S. Ramelow, R Lapkiewicz, and A. Zeilinger, Quantum imaging with undetected photons, Nature (London) 512, 409 (2014).

[31] J. Su, L. Cui, J. Li, Y. Liu, X. Li, and Z. Y. Ou, Versatile and precise quantum state engineering by using nonlinear interferometers, Opt. Express 27, 20479 (2019).

[32] J. Li, J. Su, L. Cui, T. Xie, Z. Y. Ou, and X. Li, Generation of pure-state single photons with high heralding efficiency by using a three-stage nonlinear interferometer, Appl. Phys. Lett. 116, 204002 (2020).

[33] X. Guo, N. Liu, X. Li, and Z. Y. Ou, Complete temporal mode analysis in pulse-pumped fiber-optical parametric amplifier for continuous variable entanglement generation, Opt. Express 23, 29369 (2015).

[34] X. Li, X. Ma, Z. Y. Ou, L. Yang, L. Cui, and D. Yu, Spectral study of photon pairs generated in dispersion shifted fiber with a pulsed pump, Opt. Express 16, 32 (2008).

[35] N. Liu, Y. Liu, X. Guo, L. Yang, X. Li, and Z. Y. Ou, Approaching single temporal mode operation in twin beams generated by pulse pumped high gain spontaneous four wave mixing, Opt. Express 24, 1096 (2016).

[36] J. Su, J. Li, L. Cui, X. Li, and Z. Y. Ou, Interference between two independent multi-temporal-mode thermal fields, Phys. Rev. A 99, 013838 (2019).

[37] H. De Riedmatten, V. Scarani, I. Marcikic, A. Acín, W. Tittel, H. Zbinden, and N. Gisin, Two independent photon pairs versus four-photon entangled states in parametric down conversion, J. Mod. Opt. 51, 1637 (2004).

[38] P. Sharapova, A. M. Pérez, O. V. Tikhonova, and M. V. Chekhova, Schmidt modes in the angular spectrum of bright squeezed vacuum, Phys. Rev. A 91, 043816 (2015).

[39] I. V. Dyakonov, P. R. Sharapova, T. S. Iskhakov, and G. Leuchs, Direct Schmidt number measurement of high-gain parametric down conversion, Laser Phys. Lett. 12, 065202 (2015).

[40] X. Chen, X. Li, and Z. Y. Ou, Direct temporal mode measurement of photon pairs by stimulated emission, Phys. Rev. A 101, 033838 (2020). 